\title{
Erhaltung der Landwirtschaftsflächen bedingt Priorisierung der Leistungen (Essay)
}

Robert Huber Christian Flury Michael Weber Marco Pezzatti
Eidgenössische Forschungsanstalt für Wald, Schnee und Landschaft sowie Flury und Giuliani GmbH (CH)* Flury und Giuliani $\mathrm{GmbH}(\mathrm{CH})$

webermanagement $(\mathrm{CH})$

Amt für Landschaft und Natur des Kantons Zürich, Abteilung Landwirtschaft (CH)

\author{
Erhaltung der Landwirtschaftsflächen bedingt Priorisierung der Leistungen \\ (Essay)
}

\begin{abstract}
Fruchtbare landwirtschaftliche Böden sind in der Schweiz ein knappes Gut. Mit dem Verlust dieser Flächen durch Siedlungsentwicklung, Waldeinwuchs und Ausdehnung der Naturräume geht das Produktionspotenzial der Landwirtschaft zurück, was deren Möglichkeit einschränkt, einen Beitrag zur Ernährungssicherheit in der Schweiz zu leisten. Darüber hinaus führen die Entwicklung der landwirtschaftlichen Gebäude und Infrastrukturen sowie Immissionen wie Gerüche oder Lärm vermehrt zu raumrelevanten Nutzungskonflikten. Zur Bewältigung dieser Konflikte müssen die verschiedenen Leistungen der Landwirtschaft im Raum priorisiert werden. Dazu sind die Bedürfnisse der Bevölkerung mit den Erfordernissen der Landwirtschaft und speziell der landwirtschaftlichen Produktion differenziert abzustimmen. Eine räumliche Priorisierung der Nutzungsansprüche nach Handlungsräumen kann - wie am Beispiel des Kantons Zürich gezeigt wird - Synergien fördern, Güterabwägungen in konkreten Planungsprozessen ermöglichen und so schliesslich zum Kulturlandschutz beitragen.
\end{abstract}

Keywords: land-use change, multifunctional agriculture, land sharing, spatial planning doi: $10.3188 /$ szf. 2015.0208

* Zürcherstrasse 111, CH-8903 Birmensdorf, E-Mail robert.huber@wsl.ch

G lobale Nahrungsmittelkrisen, zunehmend volatile Preise für Agrarprodukte, der fortschreitende Klimawandel und die stetige Veränderung der gesellschaftlichen Ansprüche an die Landwirtschaft und ihre Leistungen haben der Ernährungssicherheit in den letzten Jahren zu mehr Aufmerksamkeit verholfen. Die grundlegende Frage, wie sichergestellt wird, dass die Bevölkerung jederzeit Zugang zu genügender und ausgewogener Nahrung hat, ist nicht mehr nur ein Thema für Agrarexperten, sondern hat Eingang in den gesellschaftlichen und politischen Diskurs gefunden.

Im Kontext der Ernährungssicherheit und der landwirtschaftlichen Produktion kommt der Ressource Boden eine ausserordentliche Bedeutung zu. Da fruchtbare landwirtschaftliche Böden in der Schweiz knapp sind und nur bedingt ersetzt werden können, geht mit einem Verlust landwirtschaftlicher Nutzflächen automatisch das Produktionspotenzial der Landwirtschaft zurück und damit deren Möglichkeit, in der Schweiz einen Beitrag zur Ernährungssicherheit zu leisten. Der Bund versucht dem Schutz des Kulturlandes auf verschiedenen Politikebenen gerecht zu werden. Vom Raumkonzept
Schweiz und dem Raumplanungsgesetz über die Wald- und die Landwirtschaftspolitik bis hin zum Gewässerschutz und dem qualitativen Bodenschutz wird die Erhaltung des Kulturlandes thematisiert. Trotzdem schreitet der Verlust von landwirtschaftlichen Nutzflächen stetig voran.

Vor diesem Hintergrund stellt sich die Frage, wie der Schutz des Kulturlandes effektiver gestaltet werden könnte und wie der verbleibende landwirtschaftliche Boden optimal zur Produktion von Nahrungsmitteln genutzt werden kann, ohne dass die gesellschaftlichen und wirtschaftlichen Entwicklungen zu stark tangiert werden beziehungsweise unlösbare Nutzungskonflikte entstehen.

\section{Der Druck auf die landwirtschaftlichen Flächen nimmt zu}

Die Bodennutzung hat sich in den letzten 25 Jahren deutlich verändert (Abbildung 1). Während die Siedlungs- und Verkehrsflächen zwischen 1979/85 und 2004/09 um 58400 Hektaren (+23.4\%) und die bestockten Flächen um 38500 Hektaren 


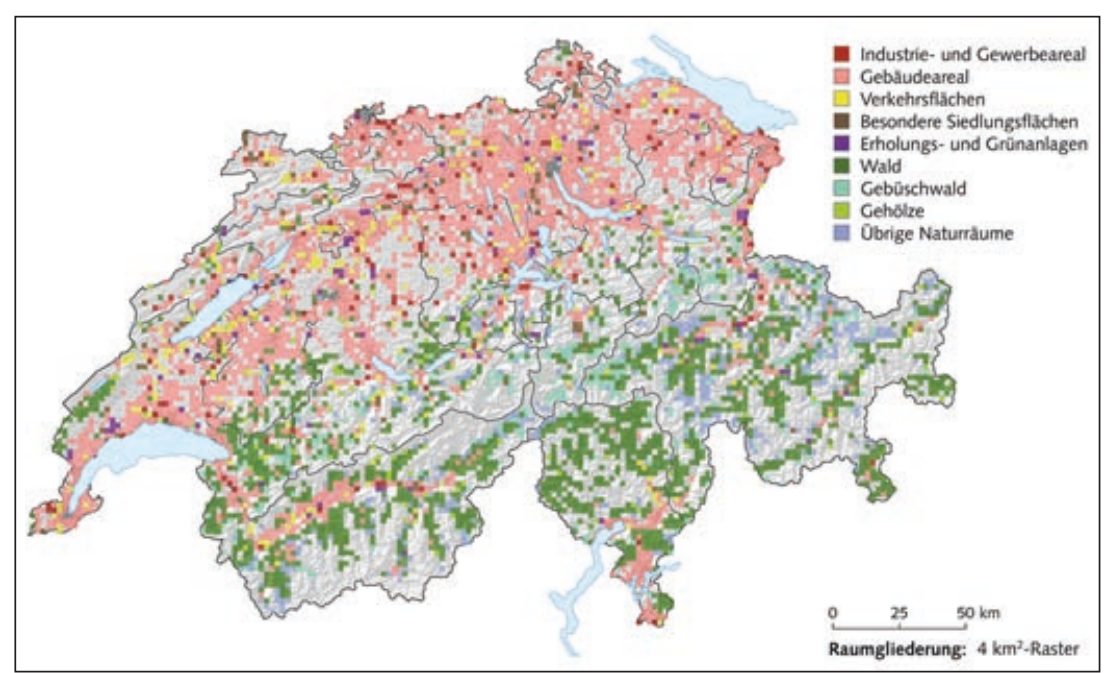

Abb 1 Neu entstandene Nutzungen auf Landwirtschaftsflächen zwischen 1985 und 2009. Farblich hervorgehoben sind diejenigen Rasterzellen, in welchen die Landwirtschaftsfläche um mindestens $1 \%$ abgenommen hat. Dargestellt ist jeweils die Nutzungsart mit der grössten Zunahme. Quelle: BFS (2015)

(+3.1\%) gewachsen sind, sind die Landwirtschaftsund Alpflächen um 85100 Hektaren geschrumpft $(-5.4 \%)$.

Dabei zeigen sich drei Trends: 1) Agronomisch wertvolle Flächen gehen im Talgebiet durch den Ausbau von Siedlungen und Infrastruktur verloren. 2) Unproduktive und schlecht erschlossene Flächen im Berg- und Sömmerungsgebiet werden aufgegeben. In der Folge werden die verbleibenden Flächen häufig intensiver genutzt. 3) Der Flächenbedarf für Ökologie und Naturschutz, insbesondere den Gewässerraum, nimmt laufend zu. Die produktiven landwirtschaftlichen Flächen kommen dadurch zusätzlich unter Druck.

Neben dem Verlust von landwirtschaftlichen Flächen durch Siedlungstätigkeit, Nutzungsaufgabe und Extensivierung nimmt der Druck auf die Landwirtschaft durch drei weitere raumrelevante Entwicklungen zu:

1. Landwirtschaftliche Zersiedlung: Die Entwicklung der landwirtschaftlichen Gebäude und Infrastrukturen stellt eine nicht unerhebliche Problematik in der Raumnutzung dar. Der landwirtschaftliche Strukturwandel und das damit verbundene Wachstum der Betriebe führen zusammen mit der fortschreitenden Spezialisierung dazu, dass immer mehr und grössere Ökonomiegebäude und Infrastrukturanlagen wie beispielsweise Gewächshäuser ausserhalb der Bauzone entstehen. Gleichzeitig muss zwischen den Interessen einer Weiternutzung von immer mehr landwirtschaftlich nicht mehr benötigten Ställen und Wohngebäuden in der Landwirtschaftszone und dem haushälterischen Umgang mit der knappen Ressource eine Güterabwägung getroffen werden.

2. Landschaftsentwicklung: Mit dem Strukturwandel geht ein Trend in Richtung grösserer Produktionseinheiten einher. Einerseits werden dadurch die Vielfalt und Gliederung der Kulturlandschaft sowie das Potenzial zur Vernetzung infrage gestellt, andererseits reduziert die Monotonisierung der Landschaft ihren Erlebniswert. Naturnahe und strukturierende Landschaftselemente gehen zugunsten der Förderung einer rationellen Bewirtschaftung und der Senkung der Produktionskosten potenziell verloren.

3. Immissionen: Die Ansprüche der zunehmend urbanen Gesellschaft an die Landwirtschaft nehmen auch ausserhalb der sichtbaren Veränderungen zu. Immissionen wie Gerüche oder Lärm werden im urbanen, aber auch im ländlichen Raum immer weniger toleriert. Für die produzierende Landwirtschaft entstehen auch dadurch raumrelevante Einschränkungen.

\section{Räumliche Priorisierung von Ansprüchen und Leistungen}

Die Betrachtung dieser Entwicklungen unterstreicht den Eindruck, dass die Landwirtschaft von allen Seiten unter Druck ist und die landwirtschaftliche Produktion zunehmend eingeschränkt wird. Die vielerorts bereits heute offenkundigen Nutzungskonflikte werden sich dadurch tendenziell verschärfen. Zur Bewältigung dieser Herausforderung steht eine Handlungsoption im Vordergrund: Die Priorisierung der verschiedenen Leistungen der Landwirtschaft im Raum. Dazu sind die Bedürfnisse der Bevölkerung mit den Erfordernissen der Landwirtschaft und speziell der landwirtschaftlichen Produktion räumlich aufeinander abzustimmen. Eine solche räumliche Priorisierung der Nutzungsansprüche kann Synergien fördern und sinnvolle Güterabwägungen ermöglichen. Diese Priorisierung geht dabei über das in der Literatur oft diskutierte Dichotom «land sharing» versus «land sparing» hinaus (Herzog \& Schüepp 2013). Es gilt auch innerhalb von multifunktionalen Landschaften Prioritäten zu setzen.

Denn das Konzept der multifunktionalen Landwirtschaft weckt den Eindruck, dass überall alles möglich ist und gleichzeitig erbracht werden kann: landwirtschaftliche Produktion, Erhaltung der Biodiversität, Schaffung von Erholungs- und Freizeitraum und vieles mehr. In der konkreten Umsetzung, insbesondere in Bezug auf den Kulturlandschutz, zeigt sich jedoch, dass eine Priorisierung notwendig ist (Huber et al 2007). In einer zunehmend urbanen Gesellschaft mit vielen Ansprüchen an Landwirtschaft und Raum führt eine Gleichbehandlung der Räume letztendlich zu einem Verlust der Heterogenität und damit zum Gegenteil des eigentlich Beabsichtigten (Lehmann \& Messerli 2007). Eine Priorisierung bedeutet aber immer auch, dass die gesellschaftlichen und landwirtschaftlichen Ansprüche gegeneinander abgewogen werden müssen. Dazu gehört auch, dass gewisse Leistungen nicht 
mehr überall erbracht werden und es Gewinner und Verlierer gibt. Partizipative Ansätze müssen entsprechend eine Priorisierung und eine Kompensation ermöglichen.

Im Kontext der Ernährungssicherheit ist eine langfristige Sicherung des Produktionsbeitrags der Landwirtschaft nur möglich, wenn räumliche Prioritäten gesetzt und andere Leistungen oder gesellschaftliche Ansprüche der Produktion untergeordnet werden. Dies gilt zuallererst für die urbanen und periurbanen Räume im Mittelland, wo sich an produktiven Standorten vielfältige und zunehmend wachsende Ansprüche in den Bereichen Freizeit und Erholung sowie Ökologie überlagern. Eine Priorisierung der unterschiedlichen Leistungen ist aber auch im ländlichen Raum und im Berggebiet angezeigt, in letzterem speziell im Kontext der Herausforderung einer fortschreitenden Waldausdehnung. Auch da ist eine Priorisierung notwendig, um knappe finanzielle Ressourcen effektiv einsetzen zu können (Huber et al 2012).

Das Potenzial einer räumlichen Differenzierung der zu erbringenden Leistungen möchten wir anhand des Projekts «Langfristige Raumentwicklungsstrategien» (LaRes) im Kanton Zürich illustrieren (Kanton Zürich 2014a). Das Beispiel eignet sich gut, da die landwirtschaftlichen Nutzflächen im Kanton Zürich in den vergangenen Jahren stark abgenommen haben und die gesellschaftlichen Ansprüche aufgrund der Bevölkerungsdichte hoch sind. Das Potenzial für Nutzungskonflikte, basierend auf den oben beschriebenen Entwicklungen, ist im Kanton Zürich entsprechend gross.

\section{Priorisierung der Nutzung in Handlungsräumen am Beispiel LaRes}

Das Raumordnungskonzept des Kantons Zürichs unterteilt die Gemeinden des Kantons in die fünf Raumtypen Naturlandschaft, Kulturlandschaft, Landschaft unter Druck, urbane Wohnlandschaft und Stadtlandschaft (Abbildung 2), deren landwirtschaftlichen Potenziale unterschiedlich charakterisiert werden können (Tabelle 1). Die Raumtypen unterscheiden sich nicht grundsätzlich in ihrer Agrarstruktur. Allerdings verlief der Agrarstrukturwandel - d.h. die Veränderung der Anzahl Landwirtschaftsbetriebe - in denjenigen Raumtypen mit höheren Flächenverlusten (Landschaft unter Druck, urbane Wohnlandschaft und Stadtlandschaft) folgerichtig etwas schneller als in Raumtypen mit weniger Flächendruck. In Anbetracht der zukünftigen Entwicklung - d.h. anhaltender Strukturwandel, zunehmender Siedlungsdruck aufgrund wirtschaftlicher und demografischer Entwicklungen und stärkere Ausrichtung der Landwirtschaftspolitik auf gesellschaftliche Bedürfnisse - ist es sinnvoll, die unterschiedlichen Raumtypen auch als unterschiedliche Handlungsräume zu betrachten.

Das heisst, dass (agrar)politische Massnahmen derart differenziert werden, dass je nach prioritären gesellschaftlichen Ansprüchen unterschiedliche Nutzungsschwerpunkte gesetzt werden können. Für die Entwicklung der Landwirtschaft und Landschaft im Kanton Zürich bedeutet dies, dass je nach Handlungsraum in Abhängigkeit von
Raumtyp

Naturlandschaft

Kulturlandschaft

Landschaft unter Druck

Urbane Wohnlandschaft

Stadtlandschaften
Charakteristika der multifunktionalen Landwirtschaft

Geringe landwirtschaftliche Aktivität, hauptsächlich aufgrund der Topografie

Bewahrte grössere und vernetzte Räume mit hoher Artenvielfalt, artenreiche Schutzgebiete

Klare Übergänge zum Siedlungsgebiet mit gestalteten Ortsrändern

Bewirtschaftung bis zu einem gewissen Grad erwünscht und zur Bewahrung der Landschaft notwendig

Handlungsraum mit dem grössten Anteil an landwirtschaftlicher Nutzfläche; Raumstruktur wesentlich von produzierender Landwirtschaft mitgeprägt

Anhaltender Strukturwandel verändert historischen Landschaftscharakter

Kompakte Siedlungen mit klaren Grenzen, aber auch traditionelle Streusiedlungsgebiete in Hügellagen

Grosse, zusammenhängende Gebiete mit landwirtschaftlicher Nutzung

Professionelle Landwirtschaft mit langfristig funktionsfähigen Strukturen

Stellenwert der Landschaft für Landwirtschaft hoch, Landschaft von Landwirtschaft geprägt, aber Druck auf offene

Landschaft (aufgrund der Siedlungsentwicklung) besonders hoch

Grenzen zwischen Siedlung und Landschaft werden unschärfer, Infrastrukturanlagen nehmen sichtbar zu

Vielfältige, oft konkurrierende Ansprüche: Arbeits- und Lebensraum, Landwirtschaft, Freizeit und Erholung, Standort für Infrastrukturanlagen

Kurze Reisezeiten in Städte und Naherholungsgebiete (Mobilität), attraktiver Wohnraum (auch in Zukunft)

Landschaftsnutzung: Erholung und Freizeitaktivität, Nutzung von landwirtschaftsnahen Angeboten, trotzdem

sinkende Akzeptanz der Landwirtschaft

Einzelne Elemente der Naturlandschaft erwünscht (Ökologie)

Überdurchschnittliche Nutzungsdichte und hohe Entwicklungsdynamik

Erholung und Freizeitaktivitäten in Landschaft/Landwirtschaft

Tab 1 Charakterisierung der fünf Handlungsräume im Kanton Zürich mit Fokus auf die Landwirtschaft. Quelle: Weber et al (2014) 


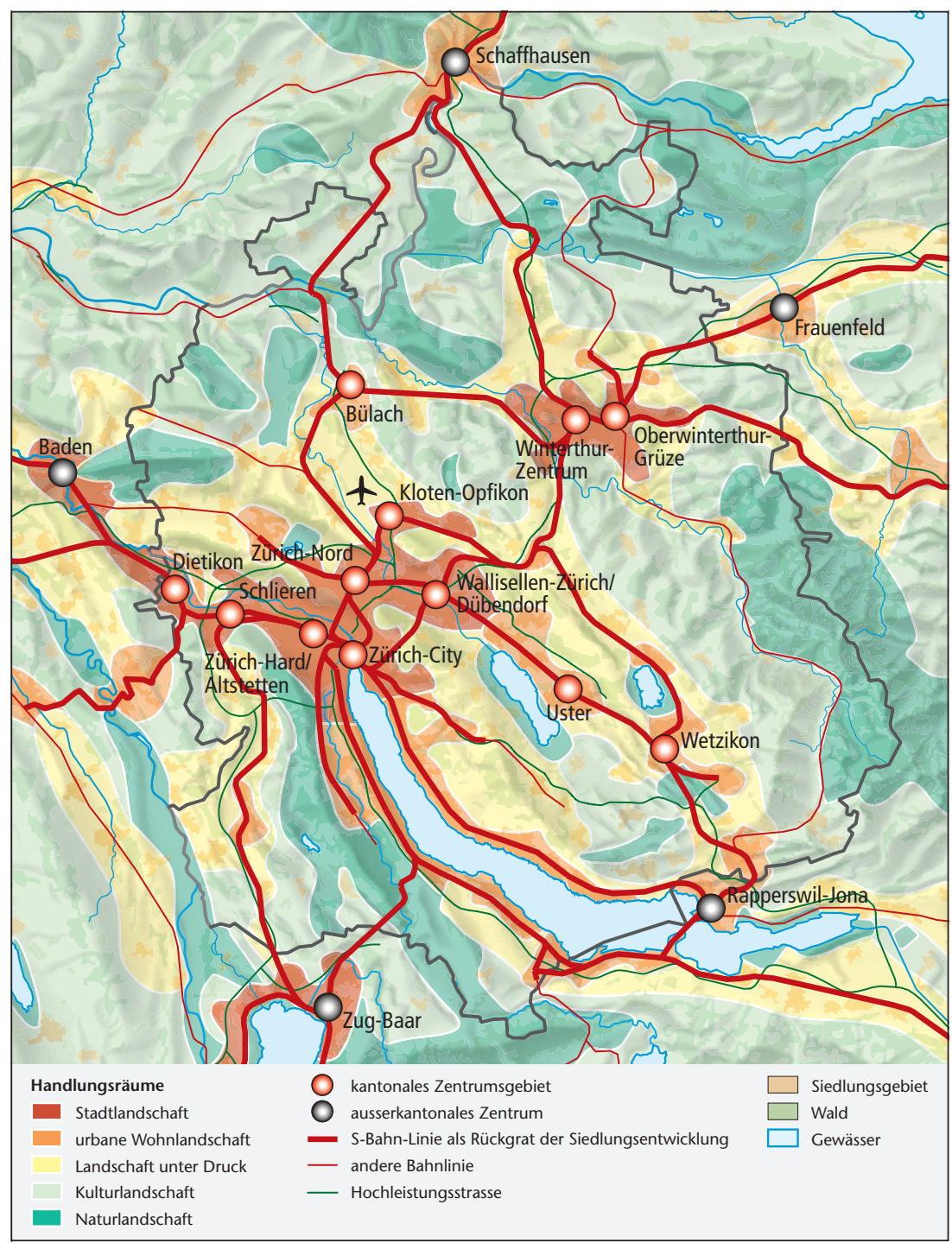

Abb 2 Handlungsräume im Grossraum Zürich. Quelle: Kanton Zürich (2014b)

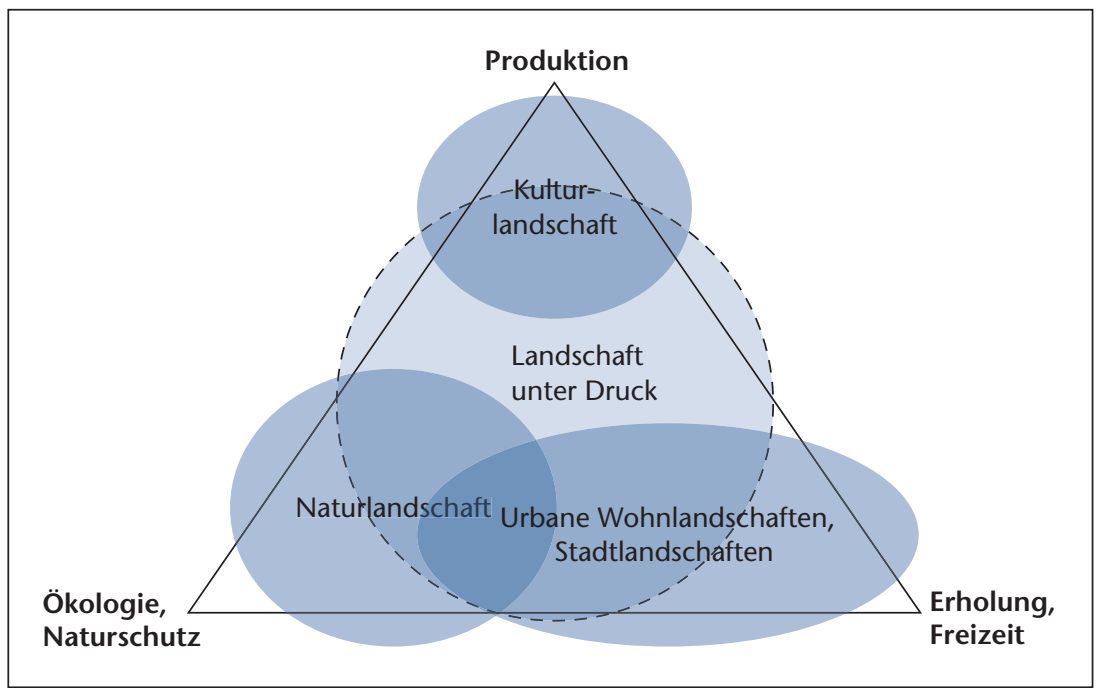

Abb 3 Priorisierung der Leistungen der Landwirtschaft nach Handlungsräumen im Kanton Zürich. Quelle: Weber et al 2014

den jeweiligen Bedürfnissen und den bestehenden Potenzialen unterschiedliche Leistungen priorisiert werden sollen (Abbildung 3).

Während in den Naturlandschaften sich die landwirtschaftliche Bewirtschaftung in den Dienst von Ökologie und Naturschutz stellen soll und dem- entsprechend Leistungen zugunsten der Landschaftsqualität und der Vernetzung von Schutzgebieten gezielt gefördert werden sollen, liegt der Fokus in den Kulturlandschaften auf der grossräumigen Entwicklung einer wettbewerbsfähigen landwirtschaftlichen Produktion. Dieser sind weiter gehende Interessen, beispielsweise der Schutz vor Geruchs- und Lärmimmissionen, unterzuordnen. Im Handlungsraum «Landschaft unter Druck» gilt es, Produktion, Naturschutz und Erholung kleinflächig und mit differenzierten Prioritäten zu entflechten. Mithilfe vermehrter, freiwilliger Partizipationsprozesse (z.B. im Rahmen der Erarbeitung von Landschaftsentwicklungskonzepten) soll dabei die Tragfähigkeit von Prioritätsentscheiden gesichert werden. In den urbanen Wohn- und in den Stadtlandschaften schliesslich steht die grossflächige Entflechtung im Vordergrund, wobei hier die Freizeit- und Erholungsnutzung Priorität haben und entsprechend Aktivitäten im Bereich der landwirtschaftlichen Diversifikation gefördert werden sollen.

Eine solche Priorisierung erlaubt es, den Schutz des Kulturlandes effektiver zu gestalten und die Nutzungskonflikte zu minimieren.

\section{Fazit}

Die Kantone haben im Rahmen des Vollzugs der Bundesvorgaben im Agrarbereich und ihrer raumplanerischen Kompetenzen genügend Spielraum für die Definition von Schwerpunkten, wie sie im Kanton Zürich gesetzt werden, und von Massnahmen, die diesen differenziert Rechnung tragen. Die Umsetzung setzt eine klare, kriterienbasierte Priorisierung der Leistungen der Landwirtschaft voraus. Die Kriterien sind regional beziehungsweise wie im Kanton Zürich nach Handlungsraum zu differenzieren. Neben den Kantonen stehen aber auch die Regionen und Gemeinden in der Verantwortung, indem sie partizipativ lokale Problemlösungen erarbeiten oder unterstützen.

Das Beispiel des Kantons Zürich zeigt, dass die räumliche Priorisierung ausgewählter Leistungen einer grundsätzlich multifunktionalen Landwirtschaft nicht nur eine konzeptionelle Idee ist, sondern in konkreten Planungsprozessen sinnvoll eingesetzt werden und als Grundlage für die Berücksichtigung von Synergien und Güterabwägungen im Kontext einer produzierenden Schweizer Landwirtschaft dienen kann. Die Definition von Handlungsräumen kann dadurch dazu beitragen, dass die Landwirtschaft einen Beitrag zur Ernährungssicherheit in der Schweiz leistet und gleichzeitig der Schutz des Kulturlandes effektiver gestaltet werden kann.

Eingereicht: 29. April 2015, akzeptiert (ohne Review): 11. Mai 2015 


\section{Literatur}

BFS (2015) Die Bodennutzung in der Schweiz. Auswertungen und Analysen. Neuenburg: Bundesamt Statistik. 64 p.

HERZOG F, SCHÜEPP C (2013) Are land sparing and land sharing real alternatives for European agricultural landscapes? Asp Appl Biol 121: 109-116.

HUBER R, BEBI P, BRINER S, BUGMANN H, BUTTLER A ET AL (2012) Waldausdehnung in zwei Regionen des Schweizer Berggebiets: eine integrative Analyse. Schweiz Z Forstwes 163: 502-511. doi: 10.3188/szf.2012.0502

HUBER R, HALLER T, WEBER M, LEHMANN B (2007) Land(wirt)schaft 2020: Was erwartet die Gesellschaft? Agrarforschung 14: 406-411.
KANTON ZÜRICH (2014A) Langfristige Raumentwicklungsstrategie Kanton Zürich. Zürich: Regierungsrat Kanton Zürich. 96 p.

KANTON ZÜRICH (2014B) Richtplan. Stand: Beschluss des Kantonsrates (Festsetzung) vom 24. März 2014. Zürich: Kanton Zürich. 204 p.

LEHMANN B, MESSERLI P (2007) The Swiss National Research Programme "Landscapes and habitats of the Alpine Arc". J Alpine Res 95 (4): 19-28.

WEBER M, SORG L, FLURY C (2014) Landwirtschaft und Landschaft im Kanton Zürich. Handlungsbedarf für die kantonale Politik. Bericht zuhanden Amt für Landschaft und Natur im Rahmen des Projekts LaRes, Zürich. Zürich: webermanagement. 40 p.

\section{Le maintien de surfaces agricoles demande d'attribuer des priorités aux prestations (essai)}

Les sols agricoles fertiles sont rares en Suisse. Avec la perte de ces surfaces par le développement du bâti, la progression de la forêt et l'extension des réserves naturelles, le potentiel de production de l'agriculture diminue, ce qui limite la possibilité de contribuer à la sécurité alimentaire en Suisse. En outre, l'expansion de bâtiments et d'infrastructures agricoles, ainsi que les perturbations olfactives ou sonores entraînent de plus en plus de conflits d'utilisation des espaces. Pour maîtriser ces conflits, il faut attribuer des priorités aux prestations de l'agriculture sur le territoire. Pour ceci, il est nécessaire d'équilibrer les besoins de la population et les exigences de l'agriculture en différenciant ses productions suivant les types d'utilisation du territoire. Des priorités spatiales pour les exigences d'utilisation peuvent encourager les synergies et permettre une appréciation judicieuse au cours des processus concrets de planification, comme le démontre l'exemple de Zurich.

\section{Protection of agricultural land needs prioritization of goods and services (essay)}

In Switzerland, productive agricultural land is scarce. Settlement development, forest encroachment and the expansion of natural habitats reduce the availability of agricultural surfaces and thus agriculture's potential to contribute to domestic food security. In addition, rural infrastructure developments as well as production immissions such as odor and noise increase land-use conflicts. To address these conflicts, society's demand for agricultural goods and services and the requirements of a productive agricultural sector must be reconciled by defining spatial areas in which goods and services provided by a multifunctional agriculture are prioritized. As shown by the example of the canton Zurich, such a spatial prioritization of agricultural goods and services allows for a consideration of synergies and trade-offs in actual planning processes to effectively protect agricultural surfaces. 